\title{
Multi-Objective Distribution Network Reconfiguration Based on Pareto Front Ranking
}

\author{
Andrea Mazza $^{1}$ - Gianfranco Chicco ${ }^{1} \cdot$ Angela Russo $^{1} \cdot$ Elena Otilia Virjoghe $^{2}$
}

Received: 30 September 2016 / Revised: 6 November 2016 / Accepted: 21 November 2016 / Published online: 1 December 2016

(C) Springer Science+Business Media Singapore 2016

\begin{abstract}
Electrical distribution system reconfiguration is frequently addressed as a multi-objective problem, typically taking into account the system losses together with other objectives, among which reliability indicators are widely used. In the multi-objective context, Pareto front analysis enables the operator handling conflicting and even noncommensurable objectives without needing the use of additional hypotheses or weights. This paper provides advances on the application of Pareto front analysis to multi-objective distribution network reconfiguration. Starting from previous results in which genetic algorithms were effectively adopted to find the best-known Pareto front, a version of the multiobjective binary particle swarm optimization (MOBPSO) customized for distribution network reconfiguration has been developed by exploiting the internal ranking of the solutions (based on a multi-criteria decision making method in the selection of the local best) and the network topology. Furthermore, the Pareto front mismatch metric (already used by the authors to compare different methods for small networks for which the complete Pareto front can be calculated) has been generalized to be used with large systems for which only the best-known Pareto front is found. Applications to a test network and to a real urban distribution network are discussed, showing the consistent superiority of the customized MOBPSO version with respect to the application of genetic algorithms and of a more classical version of the particle swarm optimization method.
\end{abstract}

\section{Angela Russo}

angela.russo@polito.it

1 Department of Energy, Politecnico di Torino, corso Duca degli Abruzzi 24, 10129 Turin, Italy

2 Faculty of Electrical Engineering, Valahia University of Targoviste, Aleea Sinaia Street 13, 130004 Targoviste, Romania
Keywords Distribution system · Reconfiguration · Binary particle swarm optimization - Multi-objective $\cdot$ Pareto front

\section{Introduction}

In the analysis of electrical distribution systems with reconfigurable structure and radial operation, for large networks the number of possible radial network configurations (calculated from the Kirchhoff's matrix tree theorem) may be so high to make exhaustive search of all the possible radial configurations practically infeasible. As such, many deterministic and probability-based methods have been exploited to find out the configurations leading to pseudo-optimal solutions for a given objective function or for multi-objective functions [1-3]. In particular, multi-objective approaches have been applied to address distribution system reconfiguration by taking into account the trade-off among conflicting objectives. Starting from early contributions [4,5], different combinations of objectives have been considered by various authors. The systems losses have been typically used in combination with other objectives such as load balancing, reliability indices, number of switching operations, branch current loading, emissions, and voltage deviations. Multi-objective reconfiguration is an effective analysis and decision-making tool for choosing the best configuration that can be operated by considering a given set of load and generation patterns.

The multiple objectives have been handled with different approaches, including: ${ }^{1}$

\footnotetext{
1 For each approach, an early paper introducing the method for optimal distribution network reconfiguration is indicated. A complete review of multi-objective methods is outside the scope of this paper.
} 
- the weighted sum of the individual objectives, using perunitized individual objectives in order to deal with noncommensurable quantities [4]; limitations on the use of the weighted sum approach are indicated in [6];

- the use of membership functions in the fuzzy domain, maximizing the fuzzy satisfaction of the optimization of the individual objectives [7];

- Pareto front analysis, based on the notion of nondominated solutions for which no other solution exists with better values for all the individual objectives [8];

- Grey correlation analysis, with the identification of problem-independent superior and inferior solutions for each objective [9].

Among these approaches, the Pareto front analysis is particularly effective because it handles non-commensurable objectives without requiring additional hypotheses or weights. The Pareto front is formed by the non-dominated solutions related to the conflicting objectives considered in multi-objective optimization. The Pareto front approach is used in many applications of stochastic optimization in different domains [10]. With reference to distribution network reconfiguration, specific techniques operating in the Pareto-based framework include the $\varepsilon$-constrained method [5] and a number of heuristics that construct and update the best-known Pareto front in an iterative way. A number of these techniques are based on genetic algorithms (GA) and evolutionary computation, such as the widely adopted Nondominated Sorting Genetic Algorithm II (NSGA II) [11] applied in some contributions [12-14], the non-dominated sorting evolutionary programming [15], and the microgenetic algorithm [16]. Further approaches use an artificial immune systems hybridized with ant colony optimization [8] or with graph theory-based techniques [17], the MultiObjective Binary Particle Swarm Optimisation (MOBPSO) [18], and a branch-and-bound technique driven by nondominance concepts [19].

Comparisons among different techniques to address multiobjective network reconfiguration have been presented in [18]. In particular, a GA procedure based on NSGA-II concepts and a version of MOBPSO have been tested, the GA procedure showing better performance. From further research carried out by the authors, the MOBPSO has been largely improved by further customizing it with respect to the specific problem addressed, providing results (shown in this paper) significantly better than the ones of the methods previously used.

Furthermore, in order to compare the results obtained from different methods using Pareto front analysis, a proper metric based on the points of the best-known Pareto front has to be exploited. Some metrics have been defined in terms of the location of the Pareto front points, such as the quality factor used in [20], depending on the number and location of the points forming the Pareto front, and the Pareto front mismatch [12], a geometrical indicator depending on the location of the Pareto front points, based on the concepts of hyper-volume calculation [21]. In order to compute the hyper-volume, a reference point is needed. In [21] the coordinates of the reference point are the worst values of the objective obtained on each dimension for the solutions found so far (including the dominated points). In this paper, the coordinates of the reference point are the worst values obtained for each dimension from the points of the best-known Pareto front found so far (excluding the dominated points). The rationale of this choice is that in the problem under analysis the worst solutions from dominated points could be very far from the region containing the points forming the Pareto front. This would generate large hyper-volumes and lead to obtain similar values for the hypervolume when the Pareto front changes. In the solution used, the region of analysis is limited to the one defined by the Pareto front points, emphasizing the differences among the solutions with different Pareto fronts.

On these bases, the specific contributions of this paper are:

1. to extend and generalize the definition of the Pareto front mismatch metric in order to apply it to real networks for which the complete Pareto front cannot be formed and only the best-known Pareto front is available;

2. to illustrate the rationale and the details of the improvements introduced in the customized MOBPSO version, in which the specific knowledge on the distribution network topology is used to modify the formulation of the solution procedure;

3. to show a set of significant and consistent results obtained on different distribution systems, including a test system and a real system.

The next sections of this paper are organized as follows. Section "Objective Functions and Constraints" summarizes the objective functions and constraints used in this paper. Section "Generalized Metrics to Compare the Results of Pareto Front-Based Optimization Methods" contains the formulation of the generalized metric used to assess the effectiveness of the Pareto front-based methods. Section "Binary PSO: Concepts and Notation" introduces the notation used for the binary PSO and illustrates the characteristics of the novel MOBPSO version used for multi-objective distribution network reconfiguration. Section "The Multi-Objective Binary PSO (MOBPSO)" contains the discussion of the results obtained in the case study applications. The last section contains the concluding remarks.

\section{Objective Functions and Constraints}

Let us consider a distribution network with $N$ nodes, $Y$ physical branches (including the ones that are kept open during 
the system operation) and $S$ supply points. The number of branches that have to remain open for obtaining a radial network is $\Lambda=Y-N+S$ [22]. The identification of the best set of $\Lambda$ branches to open (i.e., the identification of the most suitable configuration to be operated) depends on the objective function defined by the user.

In this paper, the two typical conflicting objective functions energy losses and energy not supplied (ENS) are considered for multi-objective optimization. The conflicting nature of these two objectives strongly depends on the values of failure rates of the branches. For a given time interval with $h=1, \ldots, H$ time steps of duration $\tau$ each, the energy losses in the network are computed from the energy losses $\tau R_{y h} I_{y h}^{2}$ at branch $y=1, \ldots, Y$ as:

$W_{\text {losses }}=\tau \sum_{h=1}^{H} \sum_{y=1}^{Y} R_{y h} I_{y h}^{2}$

The power losses $R_{y h} I_{y h}^{2}$ are calculated by solving at each time step $h$ a power flow based on the backward-forward sweep method [23], particularly suitable for radial distribution networks.

The ENS is a reliability indicator calculated on the basis of rated power of the network loads and duration of supply interruptions, by considering a multi-stage service restoration process after the identification of the fault [24]. By considering the set $\boldsymbol{\Theta}_{n}$ containing all the faults $\vartheta \in \boldsymbol{\Theta}_{n}$ which affect a given node $n$, the ENS formulation for node $n$ is:

$$
E N S_{n}=C_{n} \sum_{\vartheta \in \boldsymbol{\Theta}_{n}} \lambda_{\vartheta} \sum_{\xi=1}^{\Xi_{\vartheta}} \psi_{\vartheta n}^{(\xi)} \tau_{\vartheta}^{(\xi)}
$$

where $\Xi_{\vartheta}$ indicates the number of restoration stages for fault $\vartheta, \tau_{\vartheta}^{(\xi)}$ is the duration of the $\xi$ th stage of the restoration procedure, $\lambda_{\vartheta}$ is the failure rate, $C_{n}$ is the reference power of node $n$, and $\psi_{\vartheta n}^{(\xi)}$ is a Boolean variable indicating whether node $n$ is supplied during the $\xi^{\text {th }}$ stage of fault $\vartheta$, namely, $\psi_{\vartheta n}^{(\xi)}=0$ if node $n$ is supplied, otherwise $\psi_{\vartheta n}^{(\xi)}=1$. The overall reliability indicator for the entire network is calculated by summing up the contributions coming from all the nodes:

$E N S=\sum_{n=1}^{N} E N S_{n}$

The multi-objective optimization formulation is

$\min \left\{W_{\text {losses }}, E N S\right\}$

subject to the constraints referring to the thermal limits of the network branches and the lower and upper limits of the node voltage magnitude $\left(V_{\min }=0.9 \mathrm{pu}\right.$ and $\left.V_{\max }=1.1 \mathrm{pu}\right)$. Moreover, the topology constraint is handled in the solution process in order to guarantee that any network configuration formed during the solution procedure is radial [12].

\section{Generalized Metrics to Compare the Results of Pareto Front-Based Optimization Methods}

\section{Basic Concepts}

The comparison among Pareto fronts requires to set up a reference Pareto front, such that the points located on this front cannot be dominated by any point of any other Pareto front used in the analysis. The definition of the reference Pareto front is straightforward when it is possible to determine the complete Pareto front. However, in distribution network analysis, this happens only for small-size networks and microgrids, for which it is possible to generate all the radial configurations and calculate the objective functions from exhaustive search in reasonable computational time. In these cases, the metric defined in [12] can be used.

For the general case in which the network size makes exhaustive search impracticable, the complete Pareto front is not available. Hence, the extension and generalization of the Pareto front mismatch metric provided in this paper is based on using as reference Pareto front the generalized best-known Pareto front obtained in an alternative way.

In particular, probability-based optimization methods are run several times with different seeds for random number extraction, from which a certain number of best-known Pareto fronts are found for all the compared methods. All the points of the best-known Pareto fronts obtained from the compared methods are then taken simultaneously. From these points, the generalized best-known Pareto front is constructed by excluding all the dominated solutions appearing in the best-known Pareto fronts of the individual methods once considering all the points together.

Once the reference Pareto front has been defined, it is possible to calculate some metrics for comparing the Pareto front solutions. Some examples are provided below.

\section{Quality Factor}

The quality factor $Q_{F}$ metric [20] is based on the percentage of solution points found from the optimization method under analysis forming the best-known Pareto front. Let us define with $n_{F}$ the total number of points of the reference Pareto front and with $n_{0}$ the number of points of the Pareto front found by the solution method under analysis that are located on the reference Pareto front. Then, the quality factor is expressed in per cent as:

$$
Q_{F}=100 \frac{n_{0}}{n_{F}}
$$


Fig. 1 Areas used in the definition of the generalized best-known Pareto front mismatch metric. a reference area $S_{\text {ref }}$. b solution area $S_{s}$. c difference area $S_{d}$

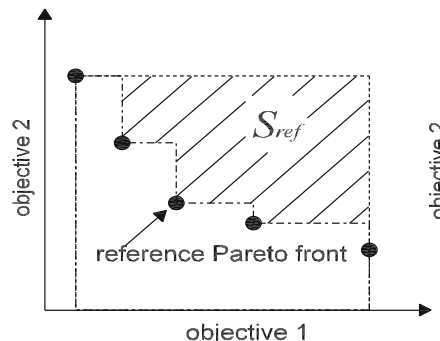

(a)

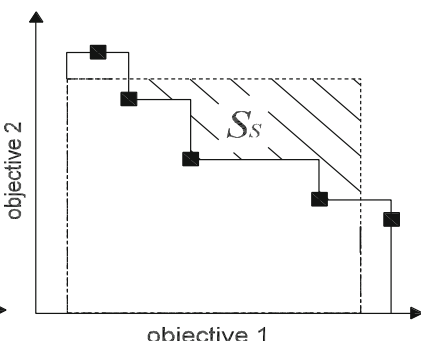

(b)

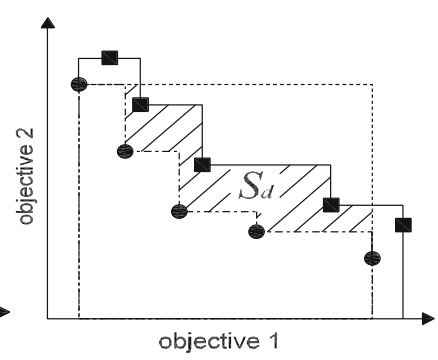

(c)

\section{Generalized Pareto Front Mismatch}

The Pareto front mismatch has been defined in [12] to introduce a more detailed metric based on geometrical considerations, taking into account not only the number of points located on the Pareto front (as in the quality factor) but also their location.

In [12] the reference Pareto front is the complete Pareto front obtained by performing exhaustive search of all the feasible network configurations. In this paper, the generalized best-known Pareto front becomes the reference Pareto front. In practice, the generalized best-known Pareto front is formed by taking the non-dominated solutions extracted from merging all the best-known Pareto fronts (the final ones obtained by repeating the optimization procedure for a given number of times with different initial seeds for random number extraction).

The pseudo-optimal solutions of the individual objective functions appearing on the reference Pareto front are used to define the upper limits for the other dimensions, hence the reference area $S_{\text {ref }}$ is calculated as indicated in Fig. 1a. Then, taking the Pareto front under analysis, the corresponding solution area $S_{S}$ (Fig. 1b) and the difference area $S_{d}=S_{\text {ref }}-S_{s}$ (Fig. 1c) are determined. The Pareto front mismatch metric associates a performance value $\gamma_{s}$ with a solution method, resulting in a given Pareto front:

$\gamma_{s}=S_{d} / S_{r e f}$

The metric (6) is used to compare different Pareto fronts, with better Pareto fronts corresponding to lower values of $\gamma_{s}$. In our case, the metric (6) has been computed for all the best-known Pareto fronts obtained by running the procedures with different seeds for random number extraction.

\section{Binary PSO: Concepts and Notation}

\section{Basic Formulation of the PSO and Related Notation}

The PSO introduced in [25] is a fast meta-heuristic method based on tracking the behavior of each element (called parti- cle) belonging to a swarm by computing the velocity of each particle.

In the optimization problem addressed in this paper, the swarm is composed of $j=1, \ldots, J$ particles, each of which represents a network configuration and contains $b=1, \ldots, B$ decision variables (binary values identifying the states of the branches, $1=$ closed and $0=$ open). The PSO is solved through an iterative process, with the generic iteration indicated with the letter $k$ and the $j=1, \ldots, J$ particles denoted as $\mathbf{x}_{j}^{(k)}=\left\{x_{j b}^{(k)}\right\}$.

Each particle changes its trajectory by following some leaders that drive the direction of variation of all the particles. In the presence of local leaders, the variation depends on the behavior of particle $j$ itself or of the nearest particles, with different possible neighborhood topologies [26,27]. The global leader affects the direction of variation of all particles, taking into account the whole history of the entire swarm.

By considering the behavior of the leaders, at iteration $k$ of the iterative process the change of configuration from $\mathbf{x}_{j}^{(k-1)}$ to $\mathbf{x}_{j}^{(k)}$ is expressed as

$\mathbf{x}_{j}^{(k)}=\mathbf{x}_{j}^{(k-1)}+\mathbf{v}_{j}^{(k)}$

in which the velocity $\mathbf{v}_{j}^{(k)}$ of particle $j$ at iteration $k$ is formulated as:

$$
\begin{aligned}
\mathbf{v}_{j}^{(k)}= & w^{(k)} \mathbf{v}_{j}^{(k-1)}+r^{\prime(k)} A_{j}^{(k)}\left(\mathbf{p}_{j}^{(k-1)}-\mathbf{x}_{j}^{(k-1)}\right)+ \\
& +r^{\prime \prime(k)} B_{j}^{(k)}\left(\tilde{\mathbf{x}}^{(k-1)}-\mathbf{x}_{j}^{(k-1)}\right)
\end{aligned}
$$

where

- $\mathbf{p}_{j}^{(k-1)}$ represents the local (or personal) best, i.e., the configuration of the particle $j$ with the best objective function found so far;

- $\tilde{\mathbf{x}}^{(k-1)}$ represents the global best, i.e., the configuration of the particle that provided the best objective function in the previous $k-1$ iterations;

- $A_{j}^{(k)}$ and $B_{j}^{(k)}$ are two learning factors that weigh the terms depending of the local and global best, respectively; 
- $r^{\prime(k)}$ and $r^{\prime \prime(k)}$ are random numbers belonging to the range $[0,1]$, applied to the whole population;

- $w^{(k)}$ is the inertia weight.

\section{Adaptive Stop Criterion}

The iterations proceed until the stop criterion is satisfied. An effective and adaptive stop criterion is to conclude the iterations when only changes in the objective function lower than a user-defined threshold $\sigma_{s}$ occur after a given number $N_{S}$ of successive iterations ${ }^{2}$ [28].

\section{Considerations on the Solutions Found}

For any heuristic method driven by probabilistic choices, there is no proof that the global optimum can be reached in a finite time. In the practical application of the discrete/binary heuristic methods like MOBPSO, the possible solutions are a countable number of points, and there is no restriction to reaching any of these points, among which the global optimum, in a finite (but not predictable) time. The evidence of this fact may be also indicated with a proof by contradiction: if there would exist a solution point that cannot be reached during the evolution of the method, the method would not be correctly formulated. However, during the execution of these heuristic methods on large systems (for which exhaustive search cannot be run because of the excessive computation time needed) there is no certainty that the global optimum has been found.

\section{Discrete and Binary PSO Representations for Single-Objective Optimization and Adaptability to the Distribution System Reconfiguration Problem}

\section{Discrete and Binary PSO Coding}

The principles of PSO operating with discrete values have been indicated in [29]. Different types of representations can be used in discrete PSO, for example based on binary entries or on integer encoding strategies [30]. The treatment of the discrete values depends on two user-defined thresholds, denoted here as $\chi$ and $E$. In practice, once the new velocity term is calculated as a real number from (8), the probability of particle evolution for each entry $b=1, \ldots, B$ of each particle $j=1, \ldots, J$ is calculated through the expression $S\left(v_{j b}^{(k)}\right)=1 /\left(1+e^{-\chi}\right)$. Then, if $S\left(v_{j b}^{(k)}\right) \leq E$ the entry $x_{j b}^{(k)}$ is not changed, otherwise it is changed. The change depends on the integer or binary representations:

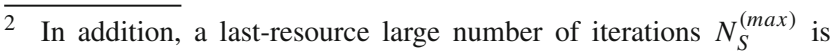
included to avoid infinite loops (generally the convergence occurs by satisfying the adaptive stop criterion before reaching $N_{S}^{(\max )}$ iterations).
}

(a) For the integer encoding strategies, rounding the velocity results to integer values is considered in [31] and the enhanced integer coded PSO method [32] has been used to minimize the total branch losses by maintaining the radial network structure and without violating the constraints. In these cases, the change can be implemented by searching for a feasible configuration represented by the integer coding used, for example adjacent to the configuration that has to be changed. In particular, in [32] the notion of local best is replaced with the so-called local optimal list (LOL), which is updated at each iteration if a good solution is found and provides a list of feasible configurations that can replace the configuration to be changed.

(b) In the binary case, the change of the entry is from 0 to 1 , or vice versa. Applying this type of change to the particles is appropriate for problems in which all the possible sequences of binary values are acceptable. However, the application of these principles to distribution system reconfiguration is not straightforward, as the mechanism of changing 0's to 1's or vice versa may create particles whose representations do not satisfy the radiality constraint [33]. This situation can be handled by generating in any case all the configurations, checking their feasibility and if infeasible generating a fictitious value of the objective function such that these configurations will be discarded in the successive part of the solution process [34]. However, in this way the generation of many infeasible solutions may occur, with a drastic increase in the solution time. Another possibility is to take the infeasible configurations and changing them in order to obtain feasible configurations [35]. In [36] a dedicated shift operator is applied to modify the chromosome structure. The Prüfer number encoding is used in [37] to generate only radial configurations for GA applications. The solution adopted in [38] and in this paper (based on binary coding) automatically generates only feasible network configurations, as described below.

\section{Binary PSO Coding Preserving Radial Configurations}

A dedicated version of the discrete PSO that benefits from the distribution network representation has been reported in [38] for the single-objective case. In this formulation, the update of the velocity vector (8) for particle $j$ at iteration $k$ is formulated as:

$\mathbf{v}_{j}^{(k)}=w^{(k)} \mathbf{v}_{j}^{(k-1)}+r^{\prime(k)} A_{j}^{(k)} \mathbf{e}_{j}^{(k)}+r^{\prime \prime(k)} B_{j}^{(k)} \mathbf{g}_{j}^{(k)}$

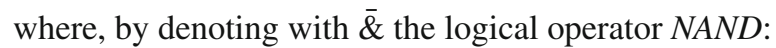

$A_{j}^{(k)}=\max \left\{0 ; f\left(\mathbf{x}_{j}^{(k-1)}\right)-f\left(\mathbf{p}_{j}^{(k-1)}\right)\right\}$ 


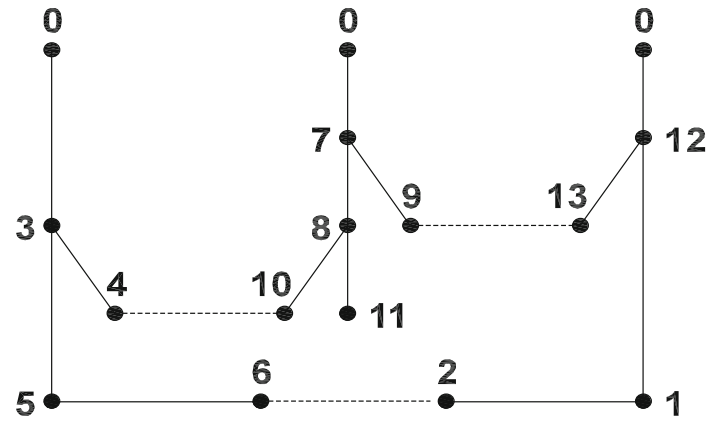

Fig. 2 Layout of the 14-node system

$B_{j}^{(k)}=\max \left\{0 ; f\left(\mathbf{x}_{j}^{(k-1)}\right)-f\left(\tilde{\mathbf{x}}^{(k-1)}\right)\right\}$

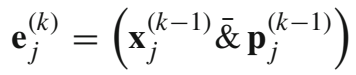

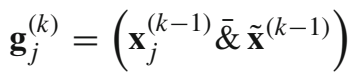

In practice, the values $A_{j}^{(k)}$ and $B_{j}^{(k)}$ indicated in (10) and (11) depend on the values of the objective function calculated in the current configuration and in the configurations corresponding to the local and global best. In addition, the velocity vector terms are not applied to each branch, but only to a subset of branches determined by using suitable logical operations.

The result of the NAND operation is modified according to the objective function values. For the addend of (9) depending on the local best, if the objective function $f\left(\mathbf{x}_{j}^{(k-1)}\right)$ is lower (i.e., better) than the objective function of the local best particle $f\left(\mathbf{p}_{j}^{(k-1)}\right)$, the non-null values coming from the $N A N D$ operation are set to zero to avoid making changes, except for the inertia weight, otherwise the difference $f\left(\mathbf{x}_{j}^{(k-1)}\right)-f\left(\mathbf{p}_{j}^{(k-1)}\right)$ is applied to modify the velocity in a way depending on the random number $r^{\prime(k)}$, as in the classical PSO. The same treatment is reserved to the addend of (9) depending on the global best.

The procedure reported in [38] continues by considering the velocity values related to the open branches of the configuration $\mathbf{x}_{j}^{(k-1)}$. These values are normalized and are then used to build the cumulative distribution function of the normalized velocity, which is processed by a biased roulette wheel procedure [39] to choose the open branch to close. The loop resulting from closing that branch is detected and, by choosing randomly the branch to open in the loop, the radial configuration is restored.

In order to provide the details of the binary PSO representation on an illustrative example, let us consider the 14-bus system [40] reported in Fig. 2, in which the nodes have been renumbered and the supply nodes have been merged into a single node (i.e., node 0 ) for purely topological reasoning. The network is composed of $Y=16$ branches, $N=14$ nodes
Table 1 Example of binary PSO operations on the 14-node network

\begin{tabular}{lrlllll}
\hline Branch from/to & & $\mathbf{x}_{j}^{(k-1)}$ & $\mathbf{p}_{j}^{(k-1)}$ & $\tilde{\mathbf{x}}_{j}^{(k-1)}$ & $\mathbf{e}_{j}^{(k)}$ & $\mathbf{g}_{j}^{(k)}$ \\
\hline 0 & 3 & 1 & 1 & 1 & 0 & 0 \\
3 & 4 & 1 & 0 & 0 & 1 & 1 \\
4 & 10 & 0 & 1 & 1 & 1 & 1 \\
8 & 10 & 1 & 1 & 1 & 0 & 0 \\
8 & 11 & 1 & 1 & 1 & 0 & 0 \\
7 & 8 & 1 & 1 & 1 & 0 & 0 \\
0 & 7 & 1 & 1 & 1 & 0 & 0 \\
7 & 9 & 1 & 1 & 0 & 0 & 1 \\
9 & 13 & 0 & 0 & 1 & 1 & 1 \\
12 & 13 & 1 & 1 & 1 & 0 & 0 \\
0 & 12 & 1 & 1 & 1 & 0 & 0 \\
1 & 12 & 1 & 1 & 1 & 0 & 0 \\
1 & 2 & 1 & 1 & 1 & 0 & 0 \\
2 & 6 & 0 & 1 & 1 & 1 & 1 \\
5 & 6 & 1 & 1 & 1 & 0 & 0 \\
3 & 5 & 1 & 0 & 0 & 1 & 1 \\
\hline
\end{tabular}

and $S=1$ supply node, so that each radial configuration has $\Lambda=3$ open branches.

By considering particle $j$ at iteration $k$, Table 1 reports for each branch the network configuration $\mathbf{x}_{j}^{(k-1)}$, the local best $\mathbf{p}_{j}^{(k-1)}$ and the global best $\tilde{\mathbf{x}}^{(k-1)}$. The operator NAND permits to find the open branches present in both configurations analyzed, but it also sets to 1 the location of the open branches present only in one of the two configurations.

\section{The Multi-Objective Binary PSO (MOBPSO)}

\section{MOBPSO Formulation}

In the multi-objective framework, a specific issue is the selection of the leaders (the local best and the global best) [41,42]. Furthermore, a quality metric is needed to understand how much a solution is better than another one [43]. This section presents a novel multi-objective version of the method reported in [38], with binary representation of the particles for multi-objective cases, solution ranking through an appropriate quality metric, and specific solutions implemented for the choice of the local best and global best by taking into account the distribution network topology.

The flow-chart is reported in Fig. 3. The initial Pareto front is formed by identifying the non-dominated points from the solutions of the initial radial configurations. The proposed procedure contains the execution of the power flow calculations, obtaining all the voltages and currents in the system. For each solution of the power flow the constraints (upper 


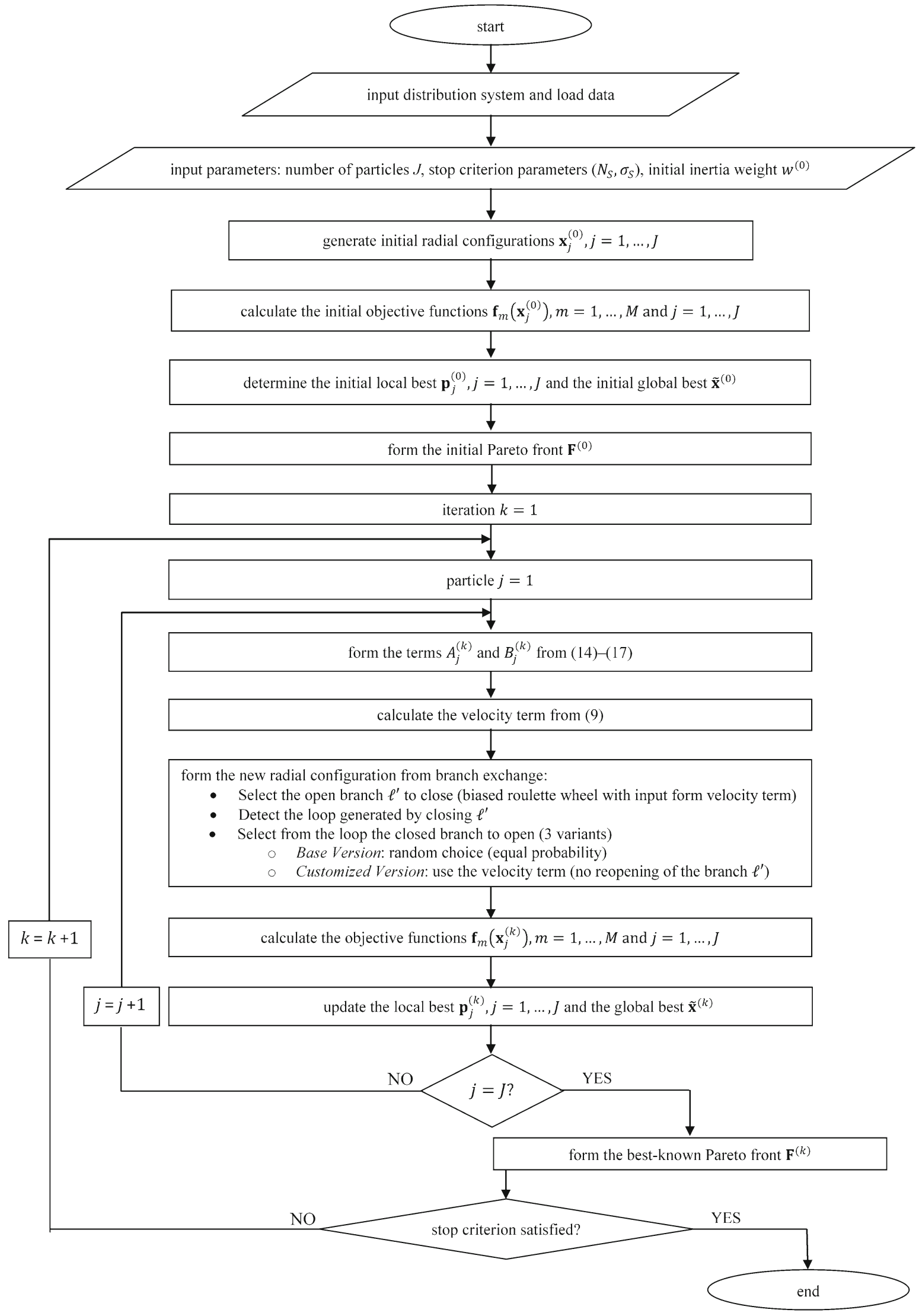

Fig. 3 Flow-chart of the proposed MOBPSO 
limits of the branch currents, and upper and lower limits of the node voltages) are checked. Each solution containing one or more violations is discarded. Thereby, only feasible solutions are processed to form the Pareto fronts. The specific aspects are addressed below, by considering two alternative versions of the method:

- Base version: it is the multi-objective application of the method composed of the equation set (9-13), with the application of the Multi-Objective Solution Ranking Method (MOSR) procedure (see Appendix) for the choice of the new population components, and the procedure to preserve the radial configuration implemented as reported in "Binary PSO Coding Preserving Radial Configurations" section. The choice of the branch to open in this case is completely random, i.e., the information coming from the velocity term is not used and all the branches forming the loop have the same probability to be open.

- Customized version: in this case the choice of the closed branch to open for keeping the network radial is made by using the velocity term (see "How to Use the Velocity Term: the Customized Version" section for details).

\section{Creation of the New Population by Applying the MOBPSO Routine: Base Version}

A formulation equivalent to (9) in the multi-objective context is presented, for $m=1, \ldots, M$ objectives.

For each particle $j=1, \ldots, J$ and each iteration $k$ the objective function values are indicated as $f_{m}\left(\mathbf{x}_{j}^{(k)}\right) \in \Re^{J, 1}$. By considering the configuration $\mathbf{x}_{j}^{(k-1)}$, the local leader $\mathbf{p}_{j}^{(k-1)}$, and the global leader $\tilde{\mathbf{x}}_{j}^{(k-1)}$ (selected inside a Leaders Archive as explained in "Update of the Global Best" section; for this reason the global leader used may change for each particle, and the subscript $j$ appears also in this case), it is possible to calculate the following quantities:

$$
\begin{aligned}
& \zeta_{m}\left(\mathbf{x}_{j}^{(k-1)}, \mathbf{p}_{j}^{(k-1)}\right) \\
& = \begin{cases}1, \text { if } f_{m}\left(\mathbf{x}_{j}^{(k-1)}\right)> & f_{m}\left(\mathbf{p}_{j}^{(k-1)}\right) \\
0, & \text { Otherwise }\end{cases} \\
& \tilde{\zeta}_{m}\left(\mathbf{x}_{j}^{(k-1)}, \tilde{\mathbf{x}}_{j}^{(k-1)}\right) \\
& = \begin{cases}1, \text { if } f_{m}\left(\mathbf{x}_{j}^{(k-1)}\right)> & f_{m}\left(\tilde{\mathbf{x}}_{j}^{(k-1)}\right) \\
0, & \text { Otherwise }\end{cases}
\end{aligned}
$$

The logical values are calculated by (14) and (15) for all the objective functions. By using the logical operation $O R$ (indicated here with $\cup$ ), it is possible to "sum" the logical values provided by (14) and (15) and to select all the configurations presenting at least one objective higher than the objective of the leaders $\mathbf{p}_{j}^{(k-1)}$ and $\tilde{\mathbf{x}}_{j}^{(k-1)}$, respectively.
Hence, the formulation of the terms $A_{j}^{(k)}$ and $B_{j}^{(k)}$ indicated in (9) is extended to the multi-objective case as follows:

$\begin{aligned} A_{j}^{(k)} & =\bigcup_{m=1}^{M} \zeta_{m}\left(\mathbf{x}_{j}^{(k-1)}, \mathbf{p}_{j}^{(k-1)}\right) \\ B_{j}^{(k)} & =\bigcup_{m=1}^{M} \tilde{\zeta}_{m}\left(\mathbf{x}_{j}^{(k-1)}, \tilde{\mathbf{x}}_{j}^{(k-1)}\right)\end{aligned}$

In practice, the single-objective maximization appearing in (10) and (11) is replaced by the $O R$ operation giving result equal to unity if at least one solution is worse than the corresponding solution of the leader considered.

\section{How to Use the Velocity Term: the Customized Version}

With the coding used here, the velocity of each element of the particle can be only either 0 or 1 . It is then not possible to apply it directly to change the network configuration. Following the approach used in [38] and [18], the velocity values referring to the open branches in $\mathbf{x}_{j}^{(k-1)}$ are used as inputs to a biased roulette wheel mechanism, for choosing the open branch $\ell^{\prime}$ which has to be closed. Then, the set $\tilde{\mathbf{L}}_{j}^{(k)} \in \mathbb{N}^{(Y, 1)}$ (having an entry equal to 1 in correspondence to the branches forming the loop) is created. The number of elements equal to 1 in the set $\tilde{\mathbf{L}}_{j}^{(k)}$ is $\tilde{L}_{i}$.

In order to restore the radial network operation, one of the branches belonging to the loop has to be chosen and open, according with the branch exchange principles [40]. In [18] the choice of this branch was random, i.e., the information about the velocity of the branches forming the loop was not used. In this paper, an improved technique based on the information about the velocity of the branches forming the loop is used.

For this purpose, a set $\tilde{\mathbf{Z}}_{j}^{(k)}$ is introduced. Its entries $\tilde{Z}_{j}$ represent the indices of the branches both belonging to the loop $\tilde{\mathbf{L}}_{j}^{(k)}$ and being open branches in $\mathbf{x}_{j}^{(k-1)}, \mathbf{p}_{j}^{(k-1)}$ and $\tilde{\mathbf{x}}^{(k-1)}$. The set $\tilde{\mathbf{Z}}_{j}^{(k)}$ is calculated by applying the $O R$ operation between the two Boolean vectors $\mathbf{e}_{j}^{(k)}$ and $\mathbf{g}_{j}^{(k)}$, (defined above), and then by applying the $A N D$ operation (indicated here with $\bigcap$ ) with the loop $\tilde{\mathbf{L}}_{j}^{(k)}$ :

$\mathbf{u}_{j}^{(k)}=\left(\mathbf{e}_{j}^{(k)} \cup \mathbf{g}_{j}^{(k)}\right) \cap \tilde{\mathbf{L}}_{j}^{(k)}$

Then, the set $\tilde{\mathbf{Z}}_{j}^{(k)}$ can be written as

$\tilde{\mathbf{Z}}_{j}^{(k)}=\hbar\left(\mathbf{u}_{j}^{(k)}\right)$

where $\hbar\left(\mathbf{u}_{j}^{(k)}\right)$ indicates the operator giving as output the positions of the elements set to 1 in the vector $\mathbf{u}_{j}^{(k)}$.

By denoting with $\oslash$ the empty set, in the customized version the formerly open branch $\ell^{\prime}$ (i.e., $\ell^{\prime} \in \tilde{\mathbf{Z}}_{j}^{(k)}$ ) cannot be 
re-opened, i.e., the set $\tilde{\mathbf{Z}}_{j}^{(k)}$ could be empty. If $\tilde{\mathbf{Z}}_{j}^{(k)}=\varnothing$ the entries of the auxiliary vector $\mathbf{q}^{(k)}$ are directly obtained as $q_{l}^{(k)}=1 / \tilde{L}$, for $l=1, \ldots, \tilde{L}$, that is, all the branches belonging to the loop have the same probability to be chosen. On the other hand, if the set $\tilde{\mathbf{Z}}_{j}^{(k)}$ is not empty (i.e., $\left.\tilde{\mathbf{Z}}_{j}^{(k)} \neq \oslash\right)$ the determination of the probability to be open for each loop's branch is more articulated. In particular, an auxiliary vector $\mathbf{q}^{(k)}$ (with $\tilde{L}$ elements) is introduced, and its entries $q_{l}^{(k)}$ are defined in such a way that the branches coming from the leaders have a higher probability to be open. At the same time, an offset $\delta>0$ is introduced ${ }^{3}$ in order to avoid null entries in the vector $\mathbf{q}^{(k)}$. Hence, for $l=1, \ldots, \tilde{L}$ :

$q_{l}^{(k)}= \begin{cases}\delta+v_{z}^{(k)}, & \text { if } z \in \tilde{\mathbf{Z}}_{j}^{(k)} \\ \delta, & \text { Otherwise }\end{cases}$

Then, the vector $\mathbf{q}^{(k)}$ is sent to the biased roulette wheel routine to choose the closed branch to open.

\section{Selection and Update of the Local Best and Global Best}

\section{Update of the Local Best}

The update of the local best $\mathbf{p}_{j}^{(k)}$, with $j=1, \ldots, J$, is performed by comparing the new configuration $\mathbf{x}_{j}^{(k)}$ with the corresponding previous local best $\mathbf{p}_{j}^{(k-1)}$. By exploiting the MOSR ranking concepts illustrated in the Appendix, if the multi-objective function in the new configuration $\mathbf{x}_{j}^{(k)}$ is better than the multi-objective function in $\mathbf{p}_{j}^{(k-1)}$, the personal best is updated, i.e., $\mathbf{p}_{j}^{(k)}=\mathbf{x}_{j}^{(k)}$, otherwise $\mathbf{p}_{j}^{(k)}=$ $\mathbf{p}_{j}^{(k-1)}$. In particular, in the application used in this paper, the comparison is carried out between two alternatives only. Thereby, the mathematical expressions becomes simpler and are expressed as follows:

$\mathbf{D}=\left[\begin{array}{l}\mathbf{p}_{j}^{(k-1)} \\ \mathbf{x}_{j}^{(k)}\end{array}\right]=\left[\begin{array}{ll}p_{1} & p_{2} \\ x_{1} & x_{2}\end{array}\right]$

Thus, it is possible to calculate both the normalized decision matrix $\mathbf{R}$ and, after having defined the weights $w_{1}$ and $w_{2}$ for the two objectives, the weighted normalized matrix $\mathbf{V}$ becomes:

$\mathbf{V}=\left[\begin{array}{ll}v_{11} & v_{12} \\ v_{21} & v_{22}\end{array}\right]=\mathbf{R}\left[\begin{array}{ll}w_{1} & 0 \\ 0 & w_{2}\end{array}\right]$

\footnotetext{
3 In order to adapt the offset $\delta$ to the velocity values under analysis, the value of $\delta$ is obtained by finding the minimum value of velocity $v_{\min }$ among the entries composing the set $\tilde{\mathbf{Z}}^{(k)}$ and then computing the value $\delta=v_{\min } / 2$.
}

$$
\begin{aligned}
& =\left[\begin{array}{ll}
\frac{p_{1}}{\sqrt{p_{1}^{2}+x_{1}^{2}}} & \frac{p_{2}}{\sqrt{p_{2}^{2}+x_{2}^{2}}} \\
\frac{x_{1}}{\sqrt{p_{1}^{2}+x_{1}^{2}}} & \frac{x_{2}}{\sqrt{p_{2}^{2}+x_{2}^{2}}}
\end{array}\right]\left[\begin{array}{ll}
w_{1} & 0 \\
0 & w_{2}
\end{array}\right] \\
& =\left[\begin{array}{cc}
w_{1} \frac{p_{1}}{\sqrt{p_{1}^{2}+x_{1}^{2}}} & w_{2} \frac{p_{2}}{\sqrt{p_{2}^{2}+x_{2}^{2}}} \\
w_{1} \frac{x_{1}}{\sqrt{p_{1}^{2}+x_{1}^{2}}} & w_{2} \frac{x_{2}}{\sqrt{p_{2}^{2}+x_{2}^{2}}}
\end{array}\right]
\end{aligned}
$$

From the entries of the matrix $\mathbf{V}$, the ideal solution $A^{+}$and the negative-ideal solution $A^{-}$are calculated as:

$$
\begin{aligned}
& A^{+}=\left[\min \left\{v_{11}, v_{21}\right\} \quad \min \left\{v_{12}, v_{22}\right\}\right]=\left[v_{1}^{+} v_{2}^{+}\right] \\
& A^{-}=\left[\max \left\{v_{11}, v_{21}\right\} \quad \max \left\{v_{12}, v_{22}\right\}\right]=\left[\begin{array}{ll}
v_{1}^{-} & v_{2}^{-}
\end{array}\right]
\end{aligned}
$$

Finally, the similarity indices of the two particles under analysis are obtained as:

$$
\begin{aligned}
C_{1}^{+} & =\frac{S_{1}^{-}}{S_{1}^{+}+S_{1}^{-}} \\
& =\frac{\sqrt{\left(v_{11}-v_{1}^{-}\right)^{2}+\left(v_{12}-v_{2}^{-}\right)^{2}}}{\sqrt{\left(v_{11}-v_{1}^{+}\right)^{2}+\left(v_{12}-v_{2}^{+}\right)^{2}}+\sqrt{\left(v_{11}-v_{1}^{-}\right)^{2}+\left(v_{12}-v_{2}^{-}\right)^{2}}} \\
C_{2}^{+} & =\frac{S_{2}^{-}}{S_{2}^{+}+S_{2}^{-}} \\
& =\frac{\sqrt{\left(v_{21}-v_{1}^{-}\right)^{2}+\left(v_{22}-v_{2}^{-}\right)^{2}}}{\sqrt{\left(v_{21}-v_{1}^{+}\right)^{2}+\left(v_{22}-v_{2}^{+}\right)^{2}}+\sqrt{\left(v_{21}-v_{1}^{-}\right)^{2}+\left(v_{22}-v_{2}^{-}\right)^{2}}}
\end{aligned}
$$

If $C_{1}^{+}>C_{2}^{+}$the local best does not change, i.e., $\mathbf{p}_{j}^{(k)}=$ $\mathbf{p}_{j}^{(k-1)}$; otherwise, $\mathbf{p}_{j}^{(k)}=\mathbf{x}_{j}^{(k)}$.

\section{Update of the Global Best}

The selection of the global best does not happen by resorting to the MOSR procedure, to avoid the possibility of obtaining a global best in which the Pareto front points are concentrated too much on the "central" locations, because of the trend of the ranking criterion to fall on the central region of the Pareto front. A classical procedure is the creation of a Leaders Archive, i.e., a set of solutions corresponding to non-dominated points, from which the global best is chosen in a random way each time the velocity of a particle has to be updated. ${ }^{4}$ In this way, it is possible to drive the evolution of the best-known Pareto front to include points located in different regions [44]. The Leaders Archive is formed by a predefined maximum number $N_{A}$ of points. If there are

\footnotetext{
4 In one of the variants adopted in [46], each time a global best has to be used, instead of extracting only one solution, two solutions are selected at random from the archive, and the one with the better hyper-volume is considered as the global best.
} 
more then $N_{A}$ candidate points, a selection procedure has to be used. A classical procedure is based on the crowding distance (the average distance of a point with respect to the two- or more-neighboring points [45]), reducing the number of points in the regions in which the points are very close to each other. A recent version [46] constructs the Leaders Archive by calculating the hyper-volume [21] obtained by using the point under analysis and the reference point, and updates the archive up to $N_{A}$ points.

In this paper, the update of the Leaders Archive occurs at the beginning of each iteration, by using the hyper-volume criterion [46]. Starting from the solution closest to the reference point for the hyper-volume calculation, each candidate point to be included in the Leaders Archive, sequentially taken from the adjacent solutions along the different dimensions, is associated with its incremental contribution to the global hyper-volume. If the number of candidate points is higher than $N_{A}$, the points in excess providing the smallest incremental contribution to the hyper-volume are not included in the Leaders Archive. At iteration $k$, for each particle $j=1, \ldots, J$ the global best $\tilde{\mathbf{x}}_{j}^{(k)}$ chosen to contribute in the formation of the new configuration $\mathbf{x}_{j}^{(k)}$ is randomly selected among the components of the Leaders Archive.

\section{Stop Criterion}

The adaptive stop criterion is the one indicated in "Adaptive Stop Criterion" section.

\section{Case Study Applications}

The multi-objective distribution system reconfiguration has been solved by using the customized NSGA-II method illustrated in [12] (denoted here as GA) and the two MOBPSO versions addressed in this paper. The optimization has been coded in Matlab ${ }^{\circledR}$. The applications have been run on the 33node network [47] modified as in [12] to add time-varying load and generation power patterns, and on a 90-node real medium voltage (MV) distribution network.

For the test network, the performance of the methods is quantified by using the Pareto front mismatch metric based on the complete Pareto front [12]. For the real MV network, the best-known Pareto front is built by using the results of the different optimization methods, and the performance is assessed by using the quality factor from [20] (see "Quality Factor" section) and the new generalized best-known Pareto front mismatch metric (see "Generalized Pareto Front Mismatch" section).

The input data used for the MOBPSO are $J=50$ particles, $N_{s}=15$ iterations and $N_{s}^{(\max )}=1000$ iterations for the stop criterion, and the inertia weight progressively changing from

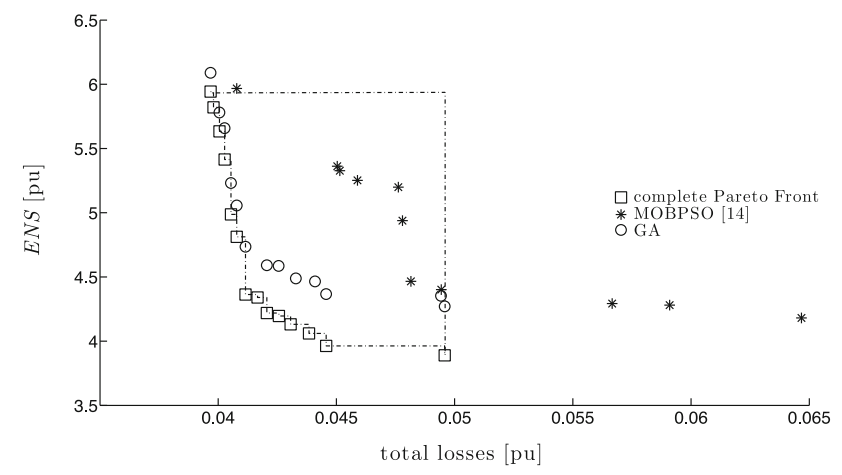

(a)

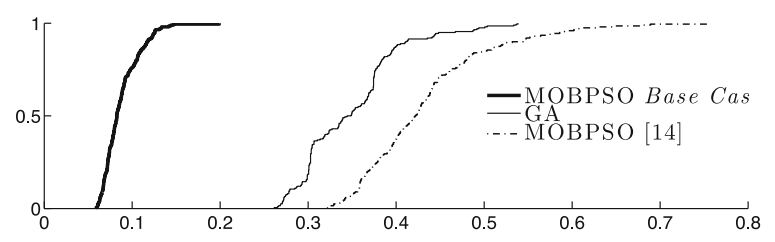

(b)

Fig. 4 Comparison among GA, MOBPSO from Mazza et al. [18] and MOBPSO base case. a Pareto fronts used to determine the Pareto front mismatch. b CDF of the Pareto front mismatch

0.9 to 0.4 during the iterations [27]. For the GA, the same parameters reported in [12] have been used.

\section{3-Node Network}

The 33-node network [47] is composed of $N=33$ nodes, $Y=37$ branches, and $S=1$ supply point, from which $\Lambda=5$ branches have to be open to obtain radial configurations. The base voltage is $12.66 \mathrm{kV}$ and the base power is 10 MVA. The voltage of the slack node is $1.05 \mathrm{pu}$. The two objectives have been computed for all the 50,751 network configurations [22] and the complete Pareto front (with 20 points) has been obtained [12]. Figure 4 a shows the complete Pareto front, and two examples of best-known Pareto front (coming from the application of GA and from the application of the version of MOPSO reported in [18])

For a more complete analysis, both the GA and the version of MOBPSO reported in [18] have been run for 200 times, with different seeds for random number extraction. The Leaders Archive for the selection of the global best has dimension $N_{A}=20$ (i.e., all the points can be randomly chosen as global best, being part of the complete Pareto front). The results shown in Fig. 4b highlight that the performance of the version of MOBPSO reported in [18] was worse than the one of the GA. Now, the results of the MOBPSO base version (in terms of reaching low values of the Pareto front mismatch, as shown from the cumulative distribution function, $\mathrm{CDF}$ ) are already considerably better than the results of the application of the GA and of the MOBPSO from [18]. 


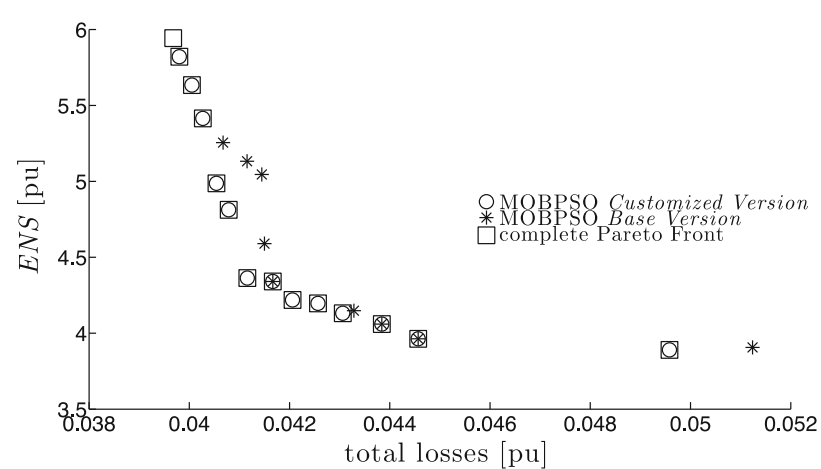

(a)

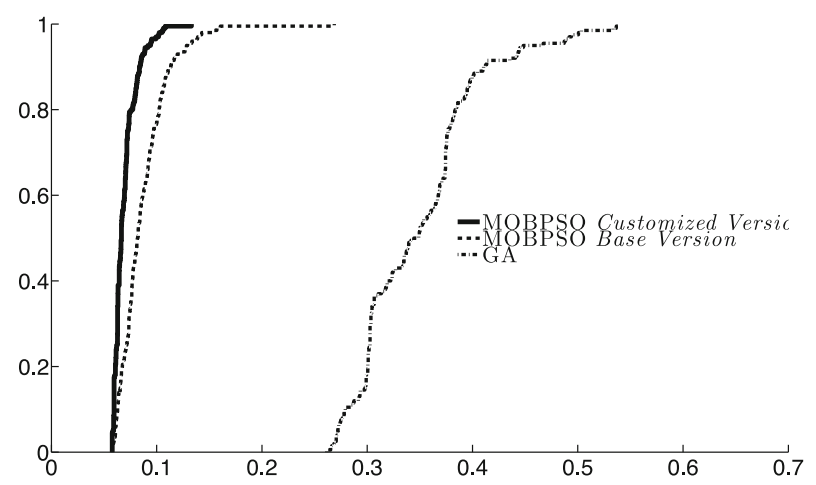

(b)

Fig. 5 Comparisons among GA and the two MOBPSO versions. a Pareto fronts used to determine the Pareto front mismatch. b CDF of the Pareto front mismatch

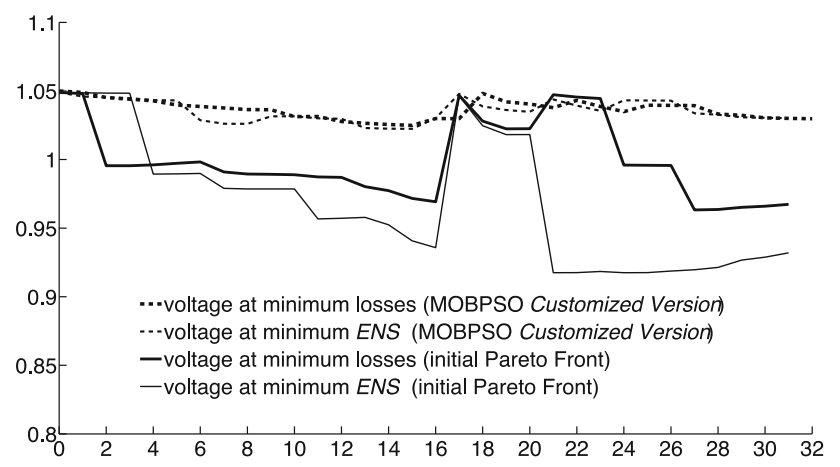

Fig. 6 Comparisons among the voltage profiles in the initial Pareto front and in the best-known Pareto front obtained with the MOBPSO customized version

Both MOBPSO versions (namely, base version and customized version) have been applied to the network. An example of best-known Pareto front for the two MOBPSO versions is shown in Fig. 5a, whereas the statistical representation (reported in Fig. 5b) shows the better performance of the MOBPSO customized version with respect to the GA and the MOBPSO base version. This means that the use of the information about the topology can effectively improve the results in the search of the complete Pareto Front.
Table 2 Computational burden for the 33-node network

\begin{tabular}{lcl}
\hline Method & Mean value [min] & SD [min] \\
\hline GA & 58.4 & 5.65 \\
MOBPSO base version & 2.4 & 0.73 \\
MOBPSO customized version & 2.6 & 0.95 \\
\hline
\end{tabular}

The voltage profiles related to the 33 -node network in four cases are shown in Fig. 6. Two cases refer to the minimum losses and minimum ENS in the initial Pareto front. The other two cases refer to the best-known Pareto front obtained by the MOBPSO customized version (Fig. 5a). Starting from the solutions belonging to the initial Pareto front, the solutions obtained in the MOBPSO customized version are characterized by remarkable improvement of the voltage profile.

Concerning the computational burden of the methods, Table 2 shows the mean value and the standard deviation of the computational times obtained by running 200 executions of the three methods on an Intel i7-4790 personal computer with CPU at $3.6 \mathrm{GHz}$ and $16 \mathrm{~GB}$ RAM. The execution times of the MOBPSO versions are relatively and significantly less than the GA version. This is mainly due to different aspects:

(i) The customized GA code contains a number of internal consistency checks to confirm that the mutation operator leads to radial configurations also by performing branch exchange when the open branch to close connects two nodes supplied by the same feeder [12]. These checks are repeated many times and increase the computation time considerably. All checks were successful, so in future version they could be skipped.

(ii) The two MOBPSO versions guarantee the radiality of the new particles formed, because the closed branches to open are chosen by using the information related to $\mathbf{x}_{j}^{(k-1)}$. Thereby no more checks are requested during the execution.

(iii) The computational time of the customized version of MOBPSO is slightly longer and more variable than the one of the base version, due to the handling of the topology. In fact, the branch exchange mechanism takes into account both the loop $\tilde{\mathbf{L}}_{j}^{(k)}$ and the open branches in $\mathbf{x}_{j}^{(k-1)}, \mathbf{p}_{j}^{(k-1)}$ and $\tilde{\mathbf{x}}^{(k-1)}$. The radiality is always guaranteed and the probability to choose open branches belonging to the leaders is higher, but the time consumption slightly increases.

\section{Real MV Urban Distribution Network}

A further comparison among the methods has been carried out on the real MV network of Fig. 7 serving an urban area. 
Fig. 7 Scheme of the real urban MV distribution network. The dashed lines correspond to the open branches in a radial configuration. A generic radial configuration is shown

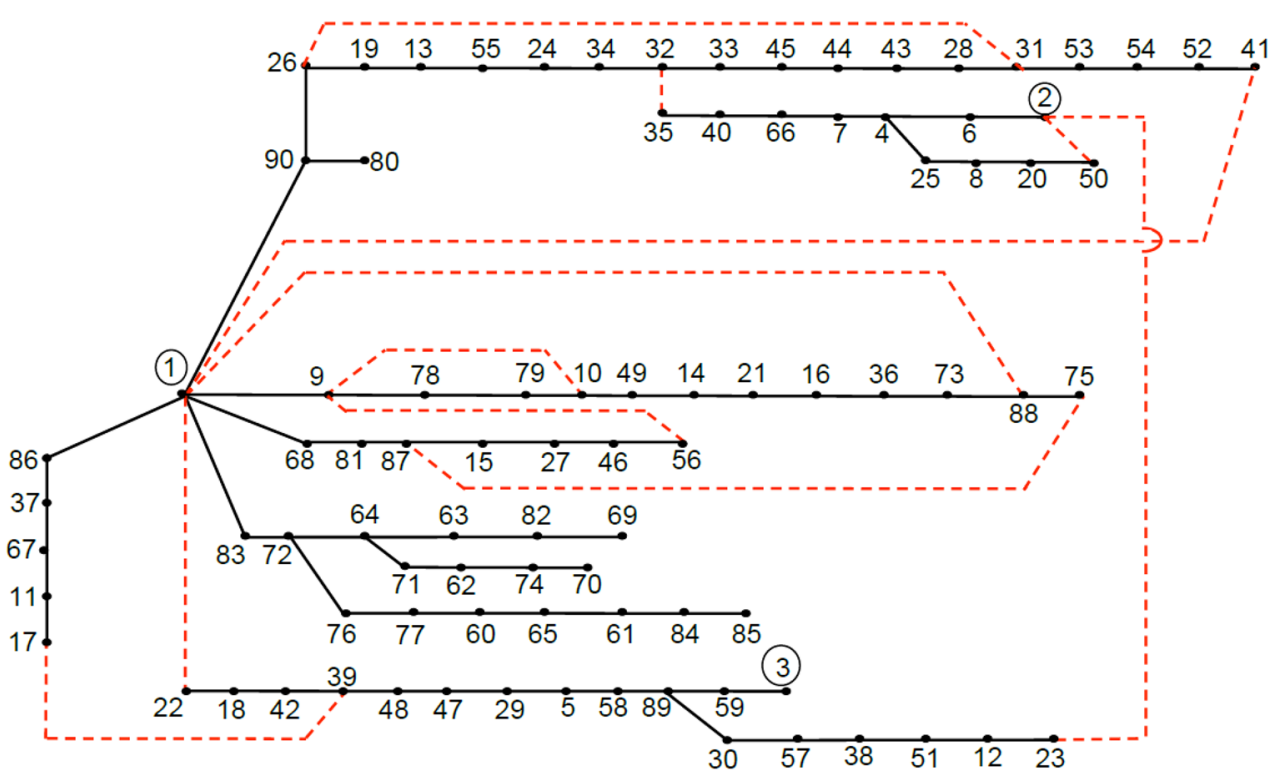

The network is composed of $N=90$ nodes, $Y=98$ branches and $S=3$ supply nodes, leading to $\Lambda=11$ branches to open to obtain radial configurations. The network base voltage is $20 \mathrm{kV}$ and the base power is 100 MVA. The supply point voltage is $1.05 \mathrm{pu}$.

The total number of radial configurations is as high as $1.176 \times 10^{10}$. Hence, it is not practicable to obtain the complete Pareto front for this network. The reference Pareto front needed to assess the performance of the methods is then constructed by combining the points of the best-known Pareto fronts obtained from the GA and the two MOBPSO versions, running each method 200 times to get a relatively higher number of best-known Pareto fronts to combine for extracting the non-dominated solutions. The Leaders Archive for the selection of the global best has dimension $N_{A}=20$. The resulting best-known Pareto front (with 67 points, Fig. 8a) is the reference Pareto front used for calculating the performance indicators. Furthermore, Fig. 8a, shows an example of best-known Pareto front obtained by applying GA and the two MOBPSO versions.

Figure $8 \mathrm{~b}$ shows that also in this case the results of the customized version are substantially better than the results of the GA application. The generalized best-known Pareto front mismatch reaches relatively low values in the best cases. This is also due to the fact that the reference Pareto front in this case is not the complete one (the values would be higher if the complete Pareto front would be available). However, for the comparison among the methods, the use of the new metric introduced is fully appropriate.

The quality factors computed for the GA and for the two MOBPSO versions are reported in Table 3. In this case, higher values correspond to better performance. The cus-

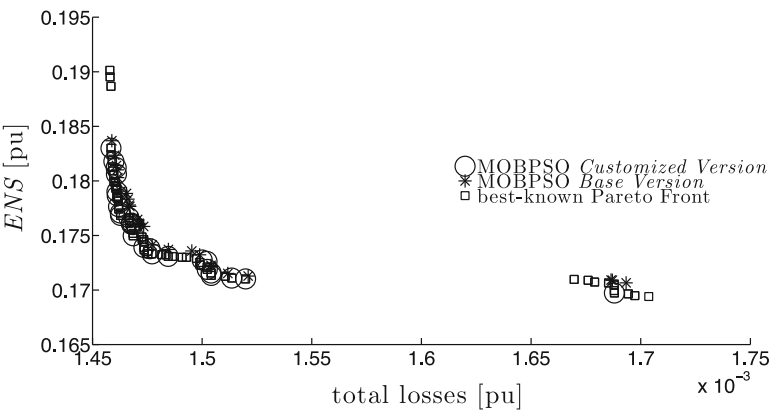

(a)

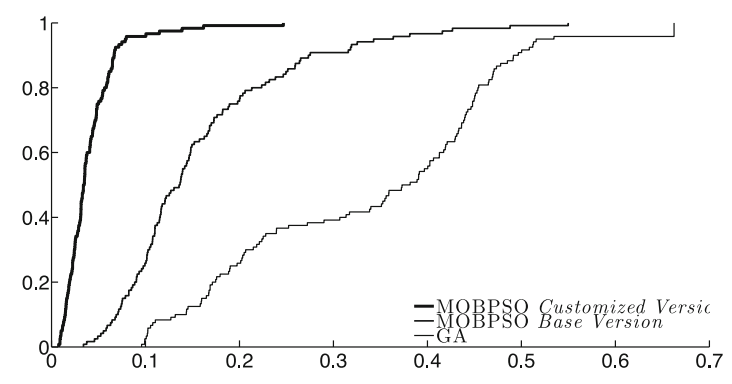

(b)

Fig. 8 Comparisons among the GA and the MOBPSO versions for the real urban MV distribution network. a Pareto fronts used to determine the Pareto front mismatch $\mathbf{b}$ CDF of the generalized best-known Pareto front mismatch

tomized version of MOBPSO largely outperforms both GA and the MOBPSO base version.

The value of the median is interesting: in fact, it highlights the most common quality factor value for both GA and base case of MOBPSO is zero, i.e., for most of the simulations the 
Table 3 Quality factor

\begin{tabular}{lccccr}
\hline Version & Max $(\%)$ & Mean $(\%)$ & SD $(\%)$ & Median $(\%)$ & 95 th percentile $(\%)$ \\
\hline GA & 10.45 & 0.6632 & 1.8198 & 0.00 & 5.22 \\
MOBPSO base version & 2.99 & 0.3234 & 0.6614 & 0.00 & 1.49 \\
MOBPSO customized version & 25.37 & 7.3010 & 5.2290 & 6.71 & 17.54 \\
\hline
\end{tabular}

Table 4 Computational burden for the real urban MV network

\begin{tabular}{lcc}
\hline Method & Mean value [min] & SD [min] \\
\hline GA & 48.2 & 18.9 \\
MOBPSO base version & 6.0 & 2.9 \\
MOBPSO customized version & 7.7 & 3.1 \\
\hline
\end{tabular}
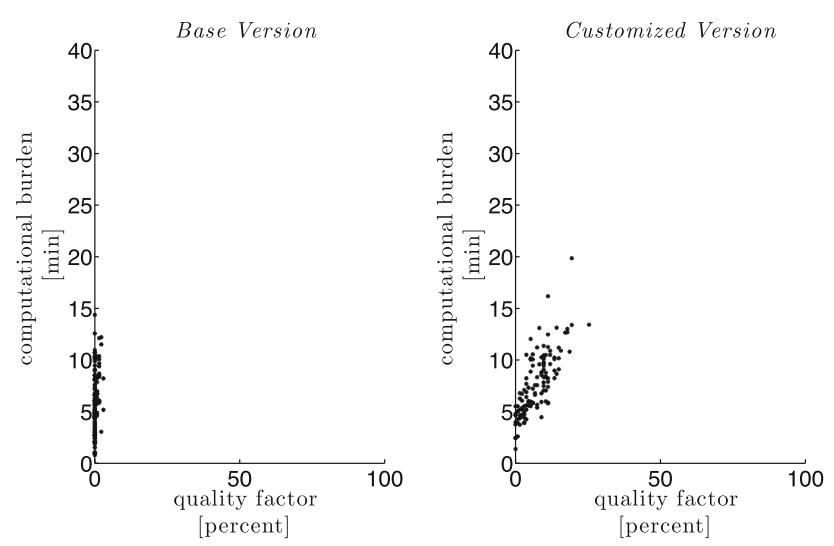

Fig. 9 Quality factor versus computational burden for the two MOBPSO versions. Higher values of the quality factor represent better solutions

solutions found do not belong to the Pareto front. In the customized version of MOBPSO versions the median is always higher than zero.

Information on the computational burden is reported in Table 4 . The order of magnitude of the computational burden for the two MOBPSO versions is comparable (mean values from about 6 minutes to about 8 minutes, obtained on an Intel i7-4790 personal computer with CPU at $3.6 \mathrm{GHz}$ and 16 GB RAM). The customized version of MOBPSO has longer computational time than the base version, justified by the handling of the topology.

A detailed representation of the relation between the performance indices and the computational burden is shown in Fig. 9 for the quality factor and in Fig. 10 for the generalized best-known Pareto front mismatch. The performance improvement for customized version is evident from the higher values of quality factor and the lower values of the generalized Pareto front mismatch, at the expense of an acceptable increase in the computational burden.

Figures 11 and 12 show the voltage profiles related to the real network. In Fig. 11, the reconfiguration at minimum
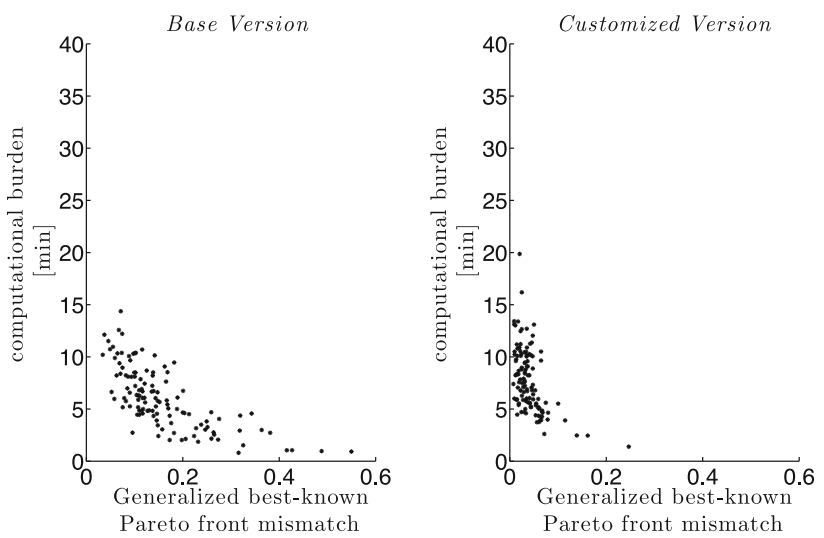

Fig. 10 Generalized best-known Pareto front mismatch versus computational burden for the two MOBPSO versions. Lower values of the generalized best-known Pareto front mismatch represent better solutions

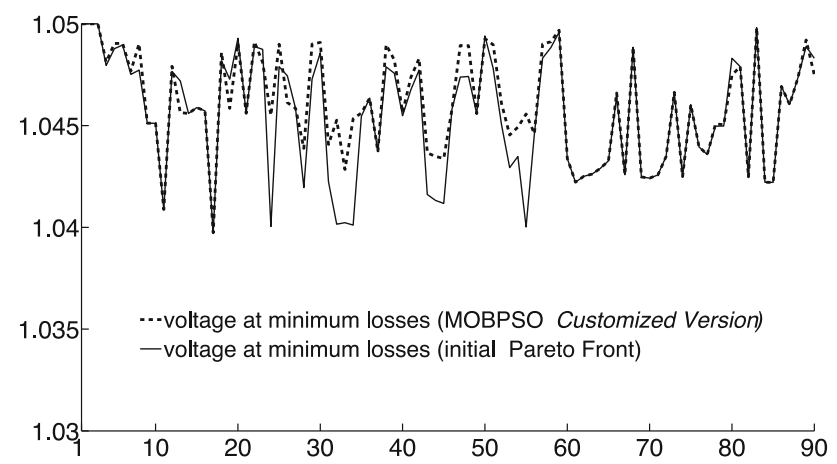

Fig. 11 Voltage profiles at minimum losses in the initial Pareto front and in the MOBPSO customized version

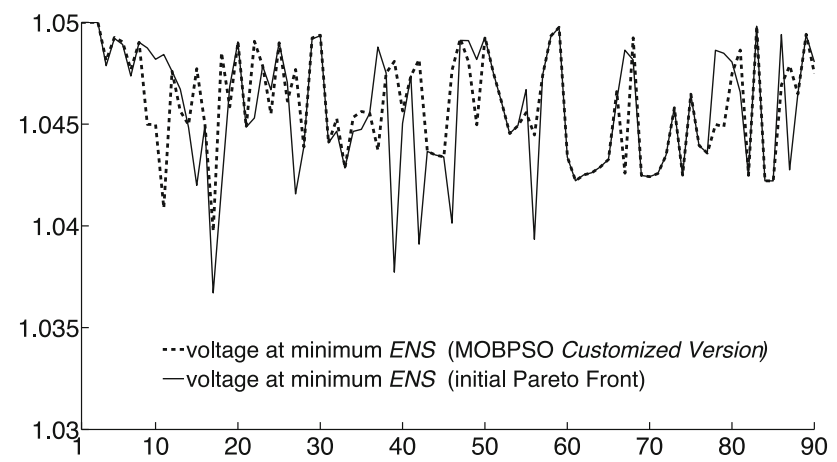

Fig. 12 Voltage profiles at minimum ENS in the initial Pareto front and in the MOBPSO customized version 
losses with the MOBPSO customized version leads to a significant improvement of the voltage profile for a large part of the network, while in the remaining part of the network (in which the open branches have not been changed with respect to the initial network) the node voltages remain the same. In Fig. 12 the optimal reconfiguration at minimum ENS carried out with the MOBPSO customized version introduces more general variations in the voltage profile. This is due to the fact that the reduction of losses is consistent with the improvement of the voltage profile, while the ENS improvement follows reliability aspects not necessarily linked with the variation of the network voltages.

\section{Conclusions}

In the research on heuristic methods, it has been often found that the customization of the algorithms to embed specific properties of the problem under analysis is successful with respect to the straightforward application of the standard version of the algorithms as general-purpose tools. Following this rationale, in this paper specific novel contributions have been presented on the performance assessment of multiobjective distribution system reconfiguration methods and on the definition of a customized MOBPSO version providing significantly better performance than previous versions of MOBPSO and GA. The generalized best-known Pareto front mismatch metric enables the user to quantify the performance of a given solution method based on the best-known Pareto front in comparison with other methods, in the application to distribution networks of any size. The MOBPSO performance has been largely improved by (i) applying internal ranking in the update of population and leaders and (ii) properly handling the elements involved in the topology variation by maintaining the radial structure. Two MOBPSO versions have been applied to test and real systems, with customized version regularly resulting as the best one. This result shows that the MOBPSO becomes more effective than the GA, raising the interest for using the customized version of MOBPSO in the light of the substantially better solutions provided. Future developments refer to the investigation of the use of further metrics in the comparison of heuristic methods and to the use of further conflicting objectives in the multi-objective reconfiguration problem.

\section{Appendix}

\section{Multi-Objective Solution Ranking (MOSR)}

In the multi-objective framework, handling the multiple objective function values indicating a single point in the solution space needs a ranking criterion acting on multi- ple dimensions, as it is not possible to use the concepts of "higher" or "lower" as in a single dimension. The ranking criterion can be based on general decision-making methods providing the ranking of two solutions (i.e., configurations, in this paper) with $M$ objective values for each solution ( $M=2$ in the examples of this paper) and identifying the winner as the top-ranked solution. Decision-making methods such as Analytic Hierarchy Process (AHP), TOPSIS and a customized decision theory-based method (details are reported in [12]) have been tested, with similar results for the various methods. The MOSR procedure used here to choose the top-ranked configuration when a decision between two alternatives occurs is based on the Technique for Order Preference by Similarity to Ideal Solution (TOPSIS) method [48]. A brief explanation of TOPSIS is provided below.

\section{Technique for Order Preference by Similarity to Ideal Solution (TOPSIS)}

A multi-criteria problem can be properly represented by a decision matrix $\mathbf{D}$, composing of $N_{P}$ rows (equal to the number of solutions under analysis) and $M$ columns (equal to the number of the objectives):

$\mathbf{D}=\left[\begin{array}{ccc}x_{11} & \cdots & x_{1 M} \\ \vdots & \ddots & \vdots \\ x_{N_{p} 1} & \cdots & x_{N_{p} M}\end{array}\right]$

The first step of the TOPSIS is a normalization process, for getting the normalized decision matrix $\mathbf{R}$ : every element of each column is divided by the norm of its column:

$r_{i j}=\frac{x_{i j}}{\sqrt{\sum_{i=1}^{N_{P}} x_{i j}^{2}}}$

The decision maker has to define the set of weights $\mathbf{w}=\left\{w_{1}, \ldots, w_{M}\right\}$,(one weight for each criterion) according to his/her opinion about them (i.e., for defining relative importance among the criteria) such that $\sum_{j=1}^{M} w_{j}=1$. Then, the weighted normalized matrix $\mathbf{V}$ (with entries $v_{i j}$, $\left.i=1 \ldots N_{P}, j=1 \ldots M\right)$ is calculated by multiplying each column of the matrix $\mathbf{R}$ by the weight $w_{j}$.

By supposing to handling a minimization problem, from the weighted normalized matrix $\mathbf{V}$ the most preferable (ideal) solution $A^{+}$and the least preferable (negative-ideal) solution $A^{-}$are calculated as:

$A^{+}=\left\{\min _{i}\left(v_{i j}\right)\right\}, \forall i=1, \ldots, N_{p}$

$A^{-}=\left\{\max _{i}\left(v_{i j}\right)\right\}, \forall i=1, \ldots, N_{p}$

Then, the indicator $S_{i}^{+}$showing the distance from the ideal solution, and the indicator $S_{i}^{-}$showing the distance 
from the negative-ideal solution, are calculated as the multidimensional Euclidean distance for every point $i=1 \ldots N_{P}$ :

$$
\begin{aligned}
& S_{i}^{+}=\sqrt{\sum_{j=1}^{M}\left(v_{i j}-v_{j}^{+}\right)^{2}} \forall i=1, \ldots, N_{p} \\
& S_{i}^{-}=\sqrt{\sum_{j=1}^{M}\left(v_{i j}-v_{j}^{-}\right)^{2}} \forall i=1, \ldots, N_{p}
\end{aligned}
$$

Finally, the relative closeness of every point to the ideal solution is computed and the best solution is found according to the following similarity index $C_{i}^{+}$:

$C_{i}^{+}=\frac{S_{i}^{-}}{S_{i}^{+}+S_{i}^{-}}, 0<C_{i}^{+}<1, \forall i=1, \ldots, N_{p}$

The solution having the maximum value of $C_{i}^{+}$is the best solution, because it is the closest one to the ideal solution.

\section{References}

1. Kalambe, S., Agnihotri, G.: Loss minimization techniques used in distribution network: bibliographical survey. Renew. Sustain. Energy Rev. 29, 184-200 (2014)

2. Mazza, A., Chicco, G., Andrei, A., Rubino, M.: Determination of the relevant periods for intraday distribution system minimum loss reconfiguration. Int. Trans. Electr. Energy Syst. 25, 1992-2023 (2015)

3. Jordehi, A.R.: Optimisation of electric distribution systems: a review. Renew. Sustain. Energy Rev. 51, 1088-1100 (2015)

4. Roytelman, I., Melnik, V., Lee, S.S.H., Lugtu, R.L.: Multi-objective feeder reconfiguration by distribution management system. IEEE Trans. Power Syst. 11, 661-667 (1996)

5. Chiang, H.D., Jean-Jumeau, V.: Optimal network reconfigurations in distribution systems. II. Solution algorithms and numerical results. IEEE Trans. Power Deliv. 5, 1568-1574 (1990)

6. Das, I., Dennis, J.E.: A closer look at drawbacks of minimizing weighted sums of objectives for Pareto set generation in multicriteria optimization problems. Struct. Optim. 14, 63-69 (1997)

7. Hsiao, Y.T., Chien, C.Y.: Multiobjective optimal feeder reconfiguration. IET Gener. Transm. Distrib 148, 333-336 (2001)

8. Ahuja, A., Das, S., Pahwa, A.: An AIS-ACO hybrid approach for multi-objective distribution system reconfiguration. IEEE Trans. Power Syst. 22, 1101-1111 (2007)

9. Tsai, M.S., Hsu, F.Y.: Application of grey correlation analysis in evolutionary programming for distribution system feeder reconfiguration. IEEE Trans. Power Syst. 25, 1126-1133 (2010)

10. Toscano, R.: Stochastic methods for hard optimization: application to robust control and fault diagnosis of industrial systems, chapter 7. In: Rigatos, G.G. (ed.) Intelligent Industrial Systems: Modeling, Automation and Adaptive Behavior. Information Science Reference, Hershey (2010)

11. Deb, K., Pratap, A., Agarwal, S., Meyarivan, T.: A fast and elitist multiobjective genetic algorithm: NSGA-II. IEEE Trans. Evol. Comput. 6, 182-197 (2002)

12. Mazza, A., Chicco, G., Russo, A.: Optimal multi-objective distribution system reconfiguration with multi criteria decision makingbased solution ranking and enhanced genetic operators. Int. J. Electr. Power Energy Syst. 54, 255-267 (2014)
13. Campoccia, A., Riva Sanseverino, E., Zizzo, G.: Considering safety issues in minimum losses reconfiguration for MV distribution networks. Eur. Trans. Electr. Power 19, 642-654 (2009)

14. Tomoiagă, B., Chindriş, M., Sumper, A., Sudria-Andreu, A., Villafafila-Robles, R.: Pareto optimal reconfiguration of power distribution systems using a genetic algorithm based on NSGA-II. Energies 6, 1439-1455 (2013)

15. Hsu, F.Y., Tsai, M.S.: A non-dominated sorting evolutionary programming algorithm for multi-objectives power distribution system feeder reconfiguration problems. Int. Trans. Electr. Energy Syst. 23, 191-213 (2013)

16. Mendoza, J.E., Lopez, M.E., Coello Coello, C.A., Lopez, E.A.: Microgenetic multiobjective reconfiguration algorithm considering power losses and reliability indices for medium voltage distribution network. IET Gener. Transm. Distrib. 3, 825-840 (2009)

17. Alonso, F.R., Oliveira, D.Q., Zambroni de Souza, A.C.: Artificial immune systems optimization approach for multiobjective distribution system reconfiguration. IEEE Trans. Power Syst. 30, 840-847 (2015)

18. Mazza, A., Chicco, G., Russo, A.: Comparison of multi-objective optimization approaches for distribution system reconfiguration. In: Proceedings of IEEE PowerTech, Grenoble (2013)

19. Guedes, L.S.M., Lisboa, A.C., Vieira, D.A.G., Saldanha, R.R.: A multiobjective heuristic for reconfiguration of the electrical radial network. IEEE Trans. Power Deliv. 28, 311-319 (2013)

20. Rabiee, M., Zandieh, M., Ramezani, P.: Bi-objective partial flexible job scheduling problem: NSGA-II, NRGA, MOGA and PAES approaches. Int. J. Prod. Res. 50, 1-16 (2012)

21. Zitzler, E., Thiele, L.: Multiobjective evolutionary algorithms: a comparative case study and the strength Pareto approach. IEEE Trans. Evol. Comput. 3, 257-271 (1999)

22. Andrei, H., Chicco, G.: Identification of the radial configurations extracted from the weakly meshed structures of electrical distribution systems. IEEE Trans. Circuits Syst. I 55, 1149-1158 (2008)

23. Shirmohammadi, D., Hong, H.W., Semlyen, A., Luo, G.X.: A compensation-based power flow method for weakly meshed distribution and transmission networks. IEEE Trans. Power Syst. 3, 753-762 (1988)

24. Carpaneto, E., Chicco, G.: Evaluation of the probability density functions of distribution system reliability indices with a characteristic functions-based approach. IEEE Trans. Power Syst. 19, 724-734 (2004)

25. Kennedy, J., Eberhart, R.: Particle Swarm Optimization. In: Proceedings of IEEE International Conference on Neural Networks, Perth, vol. 4, pp. 1942-1948 (1995)

26. Kennedy, J.: Small worlds and mega-minds: effects of neighborhood topology on particle swarm performance. In: Proceedings of the 1999 Congress on Evolutionary Computation, vol. 3, pp. 19311938 (1999)

27. Kennedy, J., Eberhart, R.: Swarm Intelligence. Morgan Kaufmann Publisher, San Francisco (2001)

28. Chicco, G., Mazza, A.: An overview of the probability-based methods for optimal electrical distribution system reconfiguration. In: Proceedings of 4th International Symposium on Electrical and Electronics Engineering, Galati (2013)

29. Kennedy, J., Eberhart, RC.: A discrete binary version of the particle swarm algorithm. In: Proceedings of Conference on Systems, Man, and Cybernetics, pp. 4104-4109 (1997)

30. Enacheanu, B., Raison, B., Caire, R., Devaux, O., Bienia, W., HadjSaid, N.: Radial network reconfiguration using genetic algorithm based on the matroid theory. IEEE Trans. Power Syst. 23, 186-195 (2008)

31. Sivanagaraju, S., Rao, J.V., Raju, P.S.: Discrete particle swarm optimization to network reconfiguration for loss reduction and load balancing. Electr. Power Compon. Syst. 36, 513-524 (2008) 
32. Wu, W.C., Tsai, M.S.: Application of enhanced integer coded particle swarm optimization for distribution system feeder reconfiguration. IEEE Trans. Power Syst. 26, 1591-1599 (2011)

33. Chang, R.F., Lu, C.N.: Feeder reconfiguration for load factor improvement. IEEE Power Eng. Soc. Winter Meet. 2, 980-984 (2002)

34. Amanulla, B., Chakrabarti, S., Singh, S.N.: Reconfiguration of power distribution systems considering reliability and power loss. IEEE Trans. Power Deliv. 27, 918-926 (2012)

35. Liu, Y., Gu, X.: Skeleton-network reconfiguration based on topological characteristics of scale-free networks and discrete particle swarm optimization. IEEE Trans. Power Syst. 22, 1267-1274 (2007)

36. Wu, W.C., Tsai, M.S., Hsu, F.Y.: A new binary coding particle swarm optimization for feeder reconfiguration. In: Proceeding of the International Conference on Intelligent Systems Applications to Power Systems (2007)

37. Hong, Y.Y., Ho, S.Y.: Determination of network configuration considering multiobjective in distribution systems using genetic algorithms. IEEE Trans. Power Syst. 20, 1062-1069 (2005)

38. Batrinu, F., Carpaneto, E., Chicco, G.: A novel particle swarm method for distribution system optimal reconfiguration. In: Proceedings of IEEE PowerTech (2005)

39. Goldberg, D.E.: Genetic Algorithms in Search, Optimization, and Machine Learning. Addison-Wesley, Reading (1968)

40. Civanlar, S., Grainger, J., Yin, H., Lee, S.: Distribution feeder reconfiguration for loss reduction. IEEE Trans. Power Deliv. 3, 1217-1223 (1988)
41. Coello, C.C., Lechuga M.S.: MOPSO: a proposal for multiple objective particle swarm optimization. In: Proceedings of the 2002 Congress on Evolutionary Computation, pp. 1051-1056 (2002)

42. Reyes-Sierra, M., Coello Coello, C.A.: Multi-objective particle swarm optimizers: a survey of the state of the art. Int. J. Comput. Intell. Res. 2, 287-308 (2006)

43. Andervazh, M.R., Olamaei, J., Haghifam, M.R.: Adaptive multi-objective distribution network reconfiguration using multiobjective discrete particles swarm optimisation algorithm and graph theory. IET Gener. Transm. Distrib. 7, 1367-1382 (2013)

44. Del Valle, Y., Venayagamoorthy, G., Mohagheghi, S., Hernandez, J.C., Harley, R.: Particle swarm optimization: basic concepts, variants and applications in power systems. IEEE Trans. Evol. Comput. 12, 171-195 (2008)

45. Coello, C., Pulido, G., Lechuga, M.: Handling multiple objectives with particle swarm optimization. IEEE Trans. Evol. Comput. 8, 256-279 (2004)

46. Nebro, A.J., Durillo, J.J., Coello, C.C.: Analysis of leader selection strategies in a multi-objective particle swarm optimizer. In: Proceedings of IEEE Congress on Evolutionary Computation, pp. 3153-3160 (2013)

47. Baran, M.E., Wu, F.F.: Network reconfiguration in distribution systems for loss reduction and load balancing. IEEE Trans. Power Deliv. 4, 1401-1407 (1989)

48. Hwang, C.L., Yoon, K.: Multiple Attribute Decision Making. Methods and Applications: A State-of-the-Art Survey. Springer, Berlin (1981) 